\title{
A SINGLE CLASSIFIER USING PRINCIPAL COMPONENTS VS MULTI-CLASSIFIER SYSTEM : IN LANDUSE-LANDCOVER CLASSIFICATION OF WORLDVIEW-2 SENSOR DATA
}

\author{
Laxmi Narayana Eeti ${ }^{\mathrm{a}, *}$, Krishna Mohan Buddhiraju ${ }^{\mathrm{a}}$, Avik Bhattacharya ${ }^{\mathrm{a}}$ \\ ${ }^{a}$ Centre of Studies in Resources Engineering, Indian Institute of Technology Bombay, Mumbai 400076, India \\ (laxmi.narayana,bkmohan,avikb)@iitb.ac.in
}

KEY WORDS: Loss in PCA, Multi-Classifier System, High Spectral-dimension, WorldView-2 image, principal components

\begin{abstract}
:
In remote sensing community, Principal Component Analysis (PCA) is widely utilized for dimensionality reduction in order to deal with high spectral-dimension data. However, dimensionality reduction through PCA results in loss of some spectral information. Analysis of an Earth-scene, based on first few principal component bands/channels, introduces error in classification, particularly since the dimensionality reduction in PCA does not consider accuracy of classification as a requirement. The present research work explores a different approach called Multi-Classifier System (MCS)/Ensemble classification to analyse high spectral-dimension satellite remote sensing data of WorldView-2 sensor. It examines the utility of MCS in landuse-landcover (LULC) classification without compromising any channel i.e. avoiding loss of information by utilizing all of the available spectral channels. It also presents a comparative study of classification results obtained by using only principal components by a single classifier and using all the original spectral channels in MCS. Comparative study of the classification results in the present work, demonstrates that utilizing all channels in MCS of five Artificial Neural Network classifiers outperforms a single Artificial Neural Network classifier that uses only first three principal components for classification process.
\end{abstract}

\section{INTRODUCTION}

Availability of more than one spectral channel in a satellite remote sensing dataset enables us to study and analyse various natural and artificial phenomena by extracting information through image analysis techniques. However, a large number of channels create high computational demand and also demand a large number of representative samples to train a classifier. Extracting enough number of representative samples/pixels is difficult in high dimensional data. In addition, there is redundant information found in more than one spectral channel which further adds to unnecessary computational demand. In such cases dimensionality reduction methods are employed to resolve the issues.

Principal Component Analysis (PCA) is widely used as dimensionality reduction technique in literature (Jolliffe, 2005, Gonzalez and Woods, 2002). It condenses most of the information spread across many channels into fewer number of channels. Variance of a data set, denoted by eigen values, decreases from first principal component to last component i.e. first principal component contains maximum amount of total variance of the data set (Byrne et al., 1980, Gonzalez and Woods, 2002). Last few components contain less variance and hence dropped in classification process. Therefore, by condensing higher number of channels into fewer number it reduces computational demand and possibly improves performance. In literature, application of PCA can be found in fields ranging from landuse-landcover mapping to face recognition (Byrne et al., 1980, Richards, 1984, Siljestrom Ribed and Moreno López, 1995, Li et al., 2008). Though PCA helps in reducing computational demands and avoids the need for larger number of representative samples, it should be noted that lower order components or components with small variance do have some discriminating information leading to loss of information (Geiger and Kubin, 2012).

Having noted that there will be loss of information by discarding some of the bands directly or through some transformation

\footnotetext{
*Corresponding author
}

like PCA, it is also true that it is difficult to estimate an unbiased covariance matrix without sufficiently large number of training samples, when there are large number of channels (Li et al., 2008, Chen, 2002, Karamizadeh et al., 2013). Naturally, the question arises, is there any other way that would enable us to avoid use of PCA dimensionality reduction technique and utilize all of the available channels for classification purpose?. We explore MCS in this context, each classifier working with the number of bands as the number of principal components, with the difference that subsets of input bands are chosen without any transformation.

A group of more than one classifier employed for classification task is called an MCS or an ensemble (Oza and Tumer, 2008). Each classifier in this group is called an ensemble member. Classifiers can be heterogeneous or homogeneous depending upon the need or information available about input data. The members are made to make error at some input values, so that each member is different from others in identification of objects. The outputs from each of these members are combined by a voting method (Van Erp et al., 2002), for final decision in classification task. The ensemble approach is widely used to improve generalization performance. Application and efficiency of MCS method in analysing remote sensing data can be found in (Giacinto et al., 2000, Han et al., 2012, Waske and Braun, 2009, Tumer and Ghosh, 1996).

In this paper, we investigate the utility of multi-classifier systems (MCS) in LULC classification of WorldView-2 sensor data without the need to perform dimensionality reduction. Since multiple classifiers are employed in MCS, available channels can be distributed among these ensemble members. A comparative study is done between classification using principal components by a single classifier and classification through a multi-classifier system. In this work a multi-classifier system is made of five artificial neural network classifiers as members. The test image is from WorldView-2 sensor that has 8 spectral channels.

In the present work, MCS gives higher classification accuracies, 
compared to conventional method of data reduction by PCA and then using first three principal components for classification. First three components are chosen based on the percentage of total variance they contain and to avoid redundancy. Overall classification accuracy and kappa coefficient produced by a single classifier utilizing principal components is 77.30 per cent and 0.73 respectively. Whereas, overall classification accuracy and kappa coefficient obtained through MCS, utilizing all channels, are 84.99 per cent and 0.82 respectively.

\section{DATASET}

Test Image in this work covers a small region in Mumbai, India. The size of the image is $615 \times 624$ pixels. It covers nine different landuse-landcover categories. The scene is acquired through WorldView-2 sensor. Spatial resolution is $1.84 \mathrm{~m}$. It captures scene in 8 multispectral channels. Figure 1 shows the false colour composite of the scene. The spectral ranges of sensor are shown in Table 1.
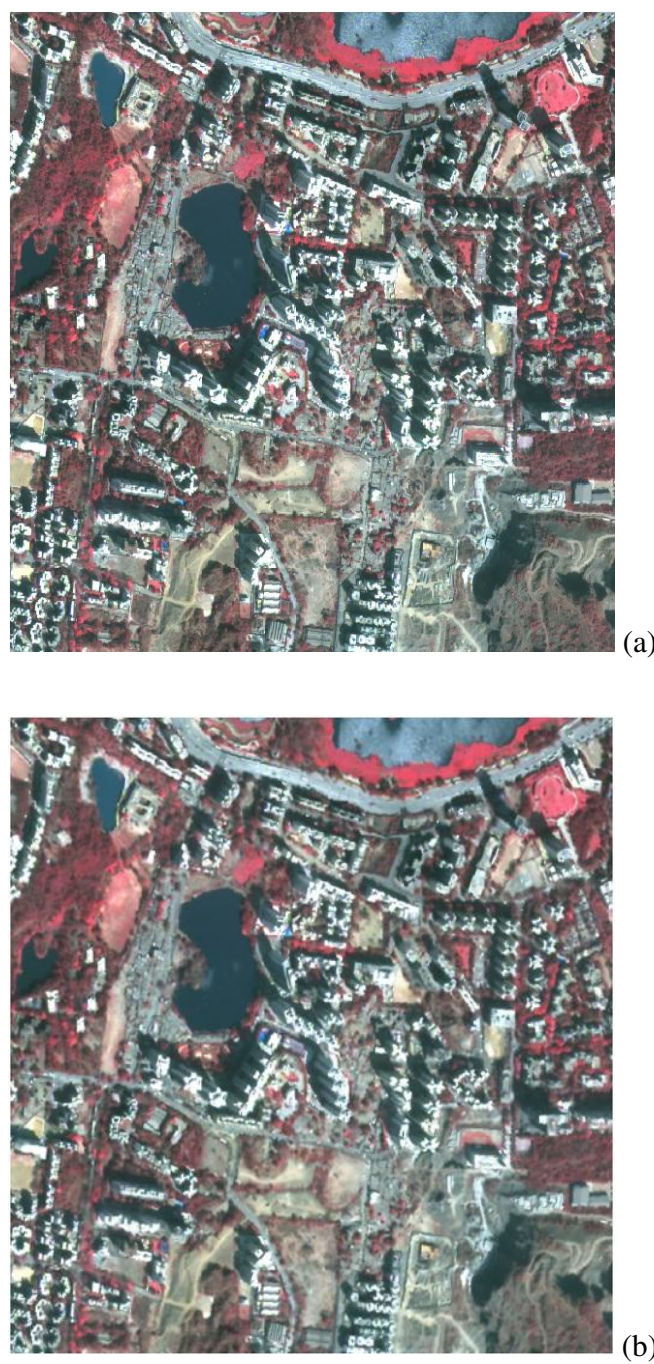

Figure 1: False colour composite of test image (a) Original (b) Smoothened by Wiener filter

\section{METHODOLOGY}

The procedure followed in data reduction, training, classification and accuracy estimation are mentioned in the following sub-

\begin{tabular}{|c|c|c|}
\hline Band 1 & Coastal & $400-450 \mathrm{~nm}$ \\
Band 2 & Blue & $450-510 \mathrm{~nm}$ \\
Band 3 & Green & $510-580 \mathrm{~nm}$ \\
Band 4 & Yellow & $585-625 \mathrm{~nm}$ \\
Band 5 & Red & $630-690 \mathrm{~nm}$ \\
Band 6 & Red Edge & $705-745 \mathrm{~nm}$ \\
Band 7 & Near IR-1 & $770-895 \mathrm{~nm}$ \\
Band 8 & Near IR-2 & $860-900 \mathrm{~nm}$ \\
\hline
\end{tabular}

Table 1: Spectral ranges in WorldView-2 sensor

sections. For training purposes a total of 6400 representative samples/pixels are used and for evaluation a total of 1846 pixels test samples are used. The test image is smoothened by Wiener filter (Lim, 1990) before extracting representative/test samples and further analysis. The Wiener filter is adaptive in nature, tailoring itself to the local image variance. Where the variance is large, it performs little smoothing. Where the variance is small, it performs more smoothing (Wiener, 1949). This approach often produces better results than linear filtering. The adaptive filter is more selective than a comparable linear filter, preserving edges and other high-frequency parts of an image. Artificial Neural Network classifiers are used in this work for classifying the image shown in Figure 1(b).Accuracy evaluation measures are in terms of overall accuracy and kappa coefficient (Richards and Richards, 1999).

\subsection{Classification by single classifier using principal compo- nents}

A variance-covariance based PCA is applied on 8 band test image of WorldView-2 to obtain principal components. The eigen values computed for each of these components are shown in Table 2. First three components are chosen for further analysis based on the fact that these three contain 98.23 per cent of total information. In literature, components that contain about 95 per cent are considered fair for image analysis requirements. Also, using more number of components defeats the purpose of dimensionality reduction. So, we considered only the first three components. Training samples are extracted from these three components and are utilized for training an artificial neural network classifier. In this work, neural network is composed of three layers- input layer, hidden layer and output layer. Nodes in each of these layers are three, fourteen and nine respectively. A well trained neural network whose generalization performance was good is chosen for classification. For classification purpose first three principal components are fed into trained classifier as inputs. Output obtained is then evaluated using test samples and a confusion matrix is generated for computing overall accuracy and kappa coefficient.

\begin{tabular}{|c|c|c|}
\hline Principal Components & Eigen Values & Variance Extracted \\
\hline PC1 & 16421.7 & 80.17 \\
PC2 & 3430.71 & 16.74 \\
PC3 & 270.215 & 1.32 \\
PC4 & 132.865 & 0.65 \\
PC5 & 88.3779 & 0.43 \\
PC6 & 69.5003 & 0.34 \\
PC7 & 37.7038 & 0.19 \\
PC8 & 31.3936 & 0.16 \\
\hline
\end{tabular}

Table 2: Eigen values and percentage information in components

\subsection{Classification using MCS}

In this work, an MCS is formed by group of five neural network classifiers as its members. So the available 8 channels are distributed among these five ensemble members. Five sets each of 
three channel-combination are constructed. The different combinations are shown in Table 3. Hence, entire information available through all the channels are utilized in MCS classifier.

\begin{tabular}{|c|c|}
\hline Ensemble Member & Channel Combination \\
\hline Member 1 & Red+Green+Blue \\
Member 2 & Red Edge+Green+Blue \\
Member 3 & IR1+Green+Blue \\
Member 4 & IR2+Green+Blue \\
Member 5 & Coastal+Green+Yellow \\
\hline
\end{tabular}

Table 3: Combination of channels as input to ensemble members

Different combinations of channels bring variety in input images for each of the ensemble members. These different combinations assure that ensemble members are trained differently and are independent in classification task to satisfy the diversity requirement in ensemble classification. Else if members behaved identically, the whole logic of ensemble classification stands null and void. The idea of constructing diverse members using different inputs is adopted in the present work and can also be found in (Pavlo et al., 2009). Diversity among ensemble members can be inferred from Table 4. It lists individual member classification accuracy along with user's and producer's accuracy of individual category.

\begin{tabular}{|c|c|c|c|c|c|}
\hline Category & Member 1 & Member 2 & Member 3 & Member 4 & Member 5 \\
\hline & \multicolumn{5}{|c|}{ Users Accuracy } \\
\hline Tree Canopy & 93.17 & 93.40 & 99.55 & 100 & 88.01 \\
Vegetation & 97.27 & 100 & 93.75 & 100 & 96.39 \\
Water & 96.86 & 86.63 & 93.70 & 86.45 & 84.58 \\
Built-up & 67.66 & 70.18 & 66.83 & 67.33 & 51.74 \\
Roadways & 53.70 & 58.86 & 66.18 & 63.81 & 52.25 \\
Hovel Rooftops & 52.85 & 78 & 64.21 & 72.28 & 67.30 \\
Shadows & 100 & 100 & 100 & 100 & 98.95 \\
Barren Land & 73.68 & 100 & 98.34 & 98.30 & 41.02 \\
Playground & 61.70 & 71.87 & 68.86 & 68.04 & 63.88 \\
\hline & \multicolumn{5}{|c|}{ Producers Accuracy } \\
\hline Tree Canopy & 99.34 & 100 & 98.45 & 100 & 98.89 \\
Vegetation & 100 & 100 & 98.13 & 100 & 100 \\
Water & 100 & 100 & 100 & 100 & 95.52 \\
Built-up & 83.95 & 94.44 & 82.09 & 82.71 & 91.35 \\
Roadways & 53.05 & 56.70 & 56.09 & 59.14 & 49.39 \\
Hovel Rooftops & 33.63 & 35.45 & 55.45 & 54.54 & 31.81 \\
Shadows & 92.02 & 68.09 & 83.43 & 61.34 & 58.28 \\
Barren Land & 49.41 & 54.70 & 70 & 68.23 & 9.411 \\
Playground & 75.65 & 100 & 100 & 100 & 80 \\
\hline \multicolumn{7}{|c|}{ Overall Accuracy } \\
\hline \multicolumn{7}{|c|}{ Kappa Coefficient } \\
\hline & 83.42 & 85.80 & 76.16 \\
\hline
\end{tabular}

Table 4: Individual member accuracy estimates

Each ensemble member is fed with a particular channel combination for training and classification purpose. The criteria for selecting a neural network and its architecture are similar as discussed previously. Each of these members produces its own classification output. For each pixel an ensemble member produces a value for each category. These values are considered as confidence measures. To combine these outputs and arrive at a final decision, the concept of confidence voting-sum rule (Van Erp et al., 2002) is adopted in the present paper. A pixel is assigned a category if total sum of confidence measure for a category exceeds other category values. The classification output is evaluated in terms of overall accuracy and kappa coefficient using test samples.

\section{RESULTS AND DISCUSSIONS}

The present study is evaluated in terms of both qualitatively and quantitatively. The classification output obtained through single classifier using principal components and that obtained by using MCS are shown in Figure 2 and Figure 3. Confusion matrices are generated in each case for evaluation of classification result. These matrices are shown in Table 5-6.

It can be inferred by comparing Table 5 and Table 6 that in each individual category, accuracy has improved using MCS. Major

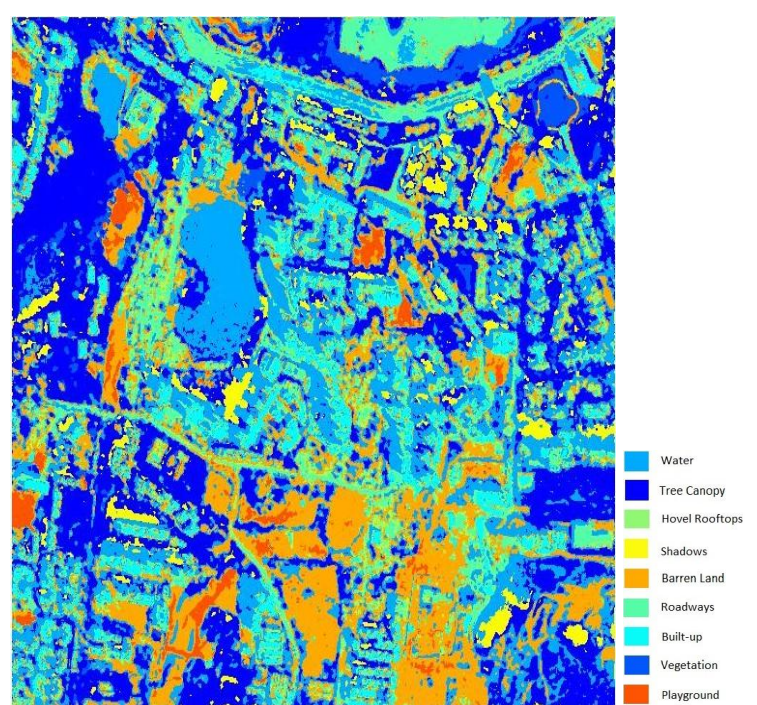

Figure 2: Classified output using single classifier

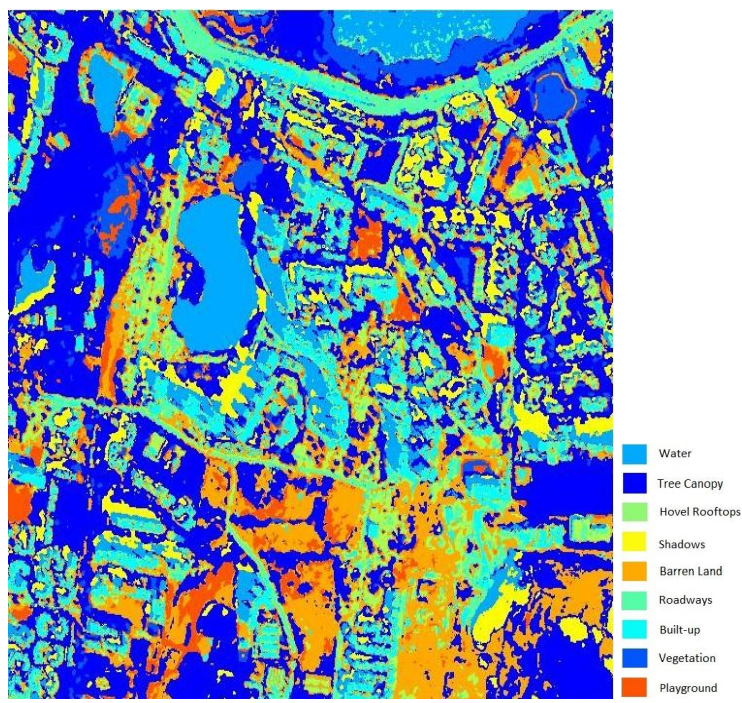

Figure 3: Classified output using ensemble(MCS)

improvements can be observed in water, roadways, hovel rooftops, shadows and considerable amount of improvement in other categories. There is improvement of about $7.69 \%$ in overall accuracy and 0.09 in kappa coefficient on comparison. The lower accuracy obtained in Table 5 might be attributed to loss of information by excluding lower order components in principal component analysis. Where as in MCS because of all the available channels were used, it produced better accuracy.

\section{CONCLUSIONS}

This paper demonstrated the efficiency and utility of Multi Classifier System (MCS) in dealing with high spectral-dimension remote sensing data such as WorldView-2 image. It has demonstrated that using MCS, one can achieve two benefits. Firstly, the need for dimensionality reduction can be avoided and secondly, higher classification accuracy can be achieved. In addition, the present paper shows that the usage of first few principal components could produce lower classification accuracy. 

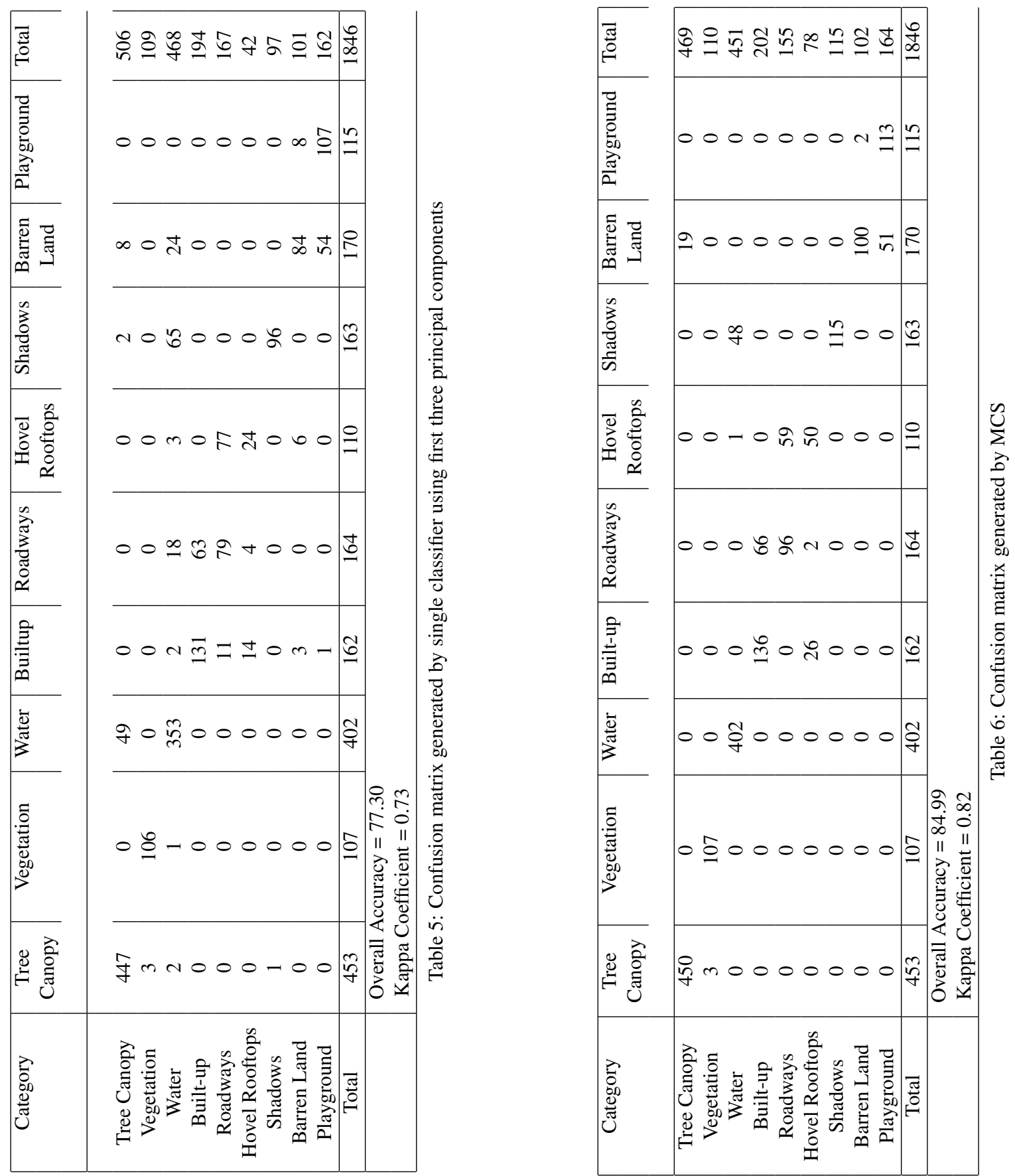


\section{REFERENCES}

Byrne, G., Crapper, P. and Mayo, K., 1980. Monitoring landcover change by principal component analysis of multitemporal landsat data. Remote Sensing of Environment 10(3), pp. $175-$ 184.

Chen, H., 2002. Principal component analysis with missing data and outliers. Electrical and Computer Engineering Department Rutgers University.

Geiger, B. C. and Kubin, G., 2012. Relative information loss in the pca. In: Information Theory Workshop (ITW), 2012 IEEE, IEEE, pp. 562-566.

Giacinto, G., Roli, F. and Bruzzone, L., 2000. Combination of neural and statistical algorithms for supervised classification of remote-sensing images. Pattern Recognition Letters 21(5), pp. 385-397.

Gonzalez, R. C. and Woods, R. E., 2002. Digital image processing.

Han, M., Zhu, X. and Yao, W., 2012. Remote sensing image classification based on neural network ensemble algorithm. Neurocomputing 78(1), pp. 133-138.

Jolliffe, I., 2005. Principal component analysis. Wiley Online Library.

Karamizadeh, S., Abdullah, S. M., Manaf, A. A., Zamani, M. and Hooman, A., 2013. An overview of principal component analysis. Journal of Signal and Information Processing 4, pp. 173.

Li, C., Diao, Y., Ma, H. and Li, Y., 2008. A statistical pca method for face recognition. In: Intelligent Information Technology Application, 2008. IITA'08. Second International Symposium on, Vol. 3, IEEE, pp. 376-380.

Lim, J. S., 1990. Two-dimensional signal and image processing. Englewood Cliffs, NJ, Prentice Hall, 1990, 710 p.

Oza, N. C. and Tumer, K., 2008. Classifier ensembles: Select real-world applications. Information Fusion 9(1), pp. 4-20.

Pavlo, A., Paulson, E., Rasin, A., Abadi, D. J., DeWitt, D. J., Madden, S. and Stonebraker, M., 2009. A comparison of approaches to large-scale data analysis. In: Proceedings of the 2009 ACM SIGMOD International Conference on Management of data, ACM, pp. 165-178.

Richards, J., 1984. Thematic mapping from multitemporal image data using the principal components transformation. Remote Sensing of Environment 16(1), pp. 35-46.

Richards, J. A. and Richards, J., 1999. Remote sensing digital image analysis. Vol. 3, Springer.

Siljestrom Ribed, P. and Moreno López, A., 1995. Monitoring burnt areas by principal components analysis of multitemporal tm data. International Journal of Remote Sensing 16(9), pp. $1577-1587$.

Tumer, K. and Ghosh, J., 1996. Analysis of decision boundaries in linearly combined neural classifiers. Pattern Recognition 29(2), pp. 341-348.

Van Erp, M., Vuurpijl, L. and Schomaker, L., 2002. An overview and comparison of voting methods for pattern recognition. In: Frontiers in Handwriting Recognition, 2002. Proceedings. Eighth International Workshop on, IEEE, pp. 195-200.

Waske, B. and Braun, M., 2009. Classifier ensembles for land cover mapping using multitemporal sar imagery. ISPRS Journal of Photogrammetry and Remote Sensing 64(5), pp. 450-457.

Wiener, N., 1949. Extrapolation, interpolation, and smoothing of stationary time series. Vol. 2, MIT press Cambridge, MA. 